\title{
Tolerancing of single point diamond turned diffractive optical elements and optical surfaces
}

\author{
R. Bittner \\ r.bittner@zeiss.de \\ Carl Zeiss AG, 73446 Oberkochen, Germany
}

Single point diamond turning is now gaining increasing importance with the production of the surfaces for different optical systems such as infrared systems, the prototype production of camera phones or head mounted displays featuring plastic lenses, or master manufacturing for the injection moulding of plastic lenses for mass products.

Tolerances which occur during single point diamond turning of aspheric surfaces and diffractive elements or during polar coordinate laser plotting of computer-generated holograms will be examined. In both cases we expect similar tolerances, because the work piece is rotated in both diamond turning and laser plotting. The objective is to understand the typical tolerances and to simulate their influence on the aberrations in the optical system. [DOI: 10.2971/jeos.2007.07028]

Keywords: Diamond turning, Laser plotting, tolerancing, optical design, diffractive optical element

\section{INTRODUCTION}

Single-point diamond turning (SPDT) has made important progress during the last twenty-five years [1]. There are significant improvements in accuracy and surface costs. With SPDT, not only aspherical surfaces but also diffractive elements on aspherical surfaces can be manufactured. Free-form optical machining is now possible. Typical surface figure errors of the SPDT process will be modelled here. In addition to SPDT, polar coordinate laser plotting [6], which produces some similar phase errors to SPDT, will be examined. Polar coordinate laser plotting allows the manufacture of diffractive structures in the form of circular rings on flat surfaces.

Very special surface figure errors occur in the manufacturing process for diffractive elements and surfaces in which the substrate rotates and the surface is produced by a special tool, in this case diamond tool or laser beam, e.g. if the axis of rotation does not match the origin of the tool's radius coordinate, then a cone-like deformation arises on the surface or in the diffractive structure.

Several approaches have been taken to the tolerancing of surface errors [2, 3]. Juergens [4] describes peculiar forms of figure errors in the SPDT process, but diffractive elements are not addressed. On the other hand, errors in the groove shape of diffractive optical elements are described by Freimann [5] for laser plotting, but the effect on the aberrations is only examined by approximation and the carrier of the diffractive element is neglected or it is a flat surface. Reichelt et al. [6] examine manufacturing errors which occur on computer-generated holograms on plane surfaces written with a polar coordinate laser plotter.

In the present paper characteristic SPDT process dependent errors of the diffractive or the aspherical elements will be analysed. Analytical formulas are derived for special errors. For some errors, e.g. tool decentre, the simultaneous effect of the diffractive and the refractive parts of the surface is significant. Suitable tools for numerical modelling have been created. Examples of different manufacturing errors are described and the resulting wavefront aberrations are shown for these error types. The effect of the surface figure errors on the diffraction efficiency and on the stray light is also important for the performance of the optical system, but it is not considered here.

\section{DESCRIPTION OF THE ASPHERICAL SURFACE AND THE DIFFRACTIVE ELEMENT}

\subsection{The sag of the aspherical surface}

For the description of the surface, the following aspherical form containing both even and odd exponents of the radial distance $h$ is used:

$$
z(h)=\frac{\rho h^{2}}{1+\sqrt{1-(1+\epsilon) \rho^{2} h^{2}}}+\sum_{m=1}^{m_{\max }} b_{m} h^{m}
$$

with

$z(h) \quad$ The sag of the aspherical surface

$\rho=1 / R$ Curvature of the surface; $R=$ Radius of the surface

$h \quad$ Radial distance

$\epsilon \quad$ Conic constant

$b_{m} \quad$ Coefficients of $h^{m}$ for $m=1, \ldots, m_{\max }$

This representation of the surface contains classic aspheres, 
conical surfaces, cones and so on and is rotationally symmetric.

\subsection{The diffractive structure}

The number of the grooves of the diffractive element, which consists of circular rings, between the axis of rotation and the radial distance $h$ is written as a polynomial with odd and even exponents:

$$
\tilde{n}(h)=\frac{1}{\lambda_{0}} \sum_{i=1}^{i_{\max }} c_{i} h^{i}
$$

with

$\tilde{n}(h)$ The number of the grooves between the axis and the radial distance $h$

$\lambda_{0} \quad$ Design wavelength

$c_{i} \quad$ Coefficients of $h^{i}$ for $i=1, \ldots, i_{\text {max }}$

$h \quad$ Radial distance

$2 \pi \tilde{n}(h)$ is also called the phase function.

For the local groove density $N(h)$, measured in the tangential plane to the surface vertex, Eq.(2) yields the following:

$$
N(h)=(\tilde{n}(h))^{\prime}=\frac{1}{\lambda_{0}} \sum_{i=1}^{i_{\max }} i c_{i} h^{i-1}
$$

\subsection{The local influence of refractive or diffractive perturbations on the OPD and their numerical treatment}

The structure of a diffractive optical element has two essential parts which can be described independently:

- the surface shape (carrier) like in Eq.(1) and

- the number of the grooves (the phase function) in Eq.(2).

During manufacture, both parts - surface shape and groove number - may display various tolerances.

A small sag difference $\Delta z$ on the surface of a lens changes the optical path difference (OPD) as follows:

$$
\triangle O P D=\frac{\Delta z \cos \phi\left(n \cos i^{\prime}-\cos i\right)}{\lambda}
$$

with

$\triangle O P D$ Change to the optical path difference normalized to $\lambda$

$\Delta z \quad$ Change to the sag parallel to the z-axis as in Eq.(1)

$\phi \quad$ Angle between surface normal and $z$-axis

$n \quad$ Index of refraction of the lens $i^{\prime} \quad$ Angle of refraction in the lens

$i \quad$ Angle of refraction in air

$\lambda \quad$ Wavelength

An error $\Delta h$ in the position of the grooves of the diffractive element at the radial distance $h$ causes:

$$
\triangle O P D=k N(h) \Delta h
$$

with

$\triangle O P D$ Change to the optical path difference normalized to $\lambda$

\section{$k \quad$ Diffraction order}

$N(h) \quad$ Groove density in lines per mm Eq.(3)

$\Delta h \quad$ Position error $h$

In principle, a local modification in the optical system in accordance with Eq.(4) or Eq.(5) will result in a change to the OPD. In the practical realization of the tolerancing of the optical system, we could work with such local modifications by adding the changes of the OPD on the particular surface to the light path. For example, in the CodeV optics design software [7] or in our in-house OASE program, we can add such local modifications to the respective surface using so-called INT files. The complete optical system can then be modelled on this basis. The INT file representation, however, also has some disadvantages: (i) First it is an approximation, whose accuracy limits must be considered for each individual case. (ii) Certain important aberrations, e.g. distortion or focal lengths which are not provided in every case, but may only be interpolated. (iii) With tolerancing of errors of diffractive elements or aspherical surfaces, the INT file should show the wavelength dependence differently according to Eq.(4) and (5), but the INT file does not. According to Eq.(5), the influence of a position error of the diffractive groove on the OPD is independent of the wavelength, while a surface deformation has a wavelength dependent influence on the OPD according to Eq.(4). For these reasons the INT file approximation was not further pursued here.

In the following, representations are generated for the various figure error functions for the surface and diffractive elements, which are accurately calculated. The modelled figure errors can be added to the surfaces either separately or together. Both the surface deformations and the defects of the diffractive element for the various tolerancing problems arising can be accurately simulated by applying a suitable surface and a suitable diffractive element containing the tolerances. The effect on the optical system is analysed by means of ray tracing.

\section{SPECIAL TOLERANCES WITH DIAMOND TURNING AND LASER PLOTTING}

With diamond turning, deformations of the generated surface may arise, which are very typical of the production process [4]. Similar errors occur with polar coordinate laser plot- 
ting, but the substrate is mostly plane here [6]. The following sources of error are definitely present, but there may be some others:

- Decentring between tool and rotation axis

- Thermal effects in production

- Deformations caused by centripetal force

- Tilting the tool's moving direction

- Uneven cutting tool wear

- Mounting stress

- Vibrations of the spindle

- Vertical spot positioning error in laser plotting

The various sources of error cause different deviations in the surface and the grooves. In this section we will model the effect of most of them. Some sources of error can simultaneously produce deviations of the surface and of the groove position of the diffractive element and therefore these deviations must be modelled together.

\subsection{Lens system example for the tolerances}

As a sample system, an IR imager with a focal length of $67.72 \mathrm{~mm}$ is used (Figure 1, Table 1). The imager consists of the stop surface, two lenses and a window in front of the detector. The diffractive element is situated on surface 3 , which is spherical here. The diffractive surface 3 is diamond turned and the refractive surfaces 2,5 and 6 also. So this example contains typical effects of diamond turned surfaces and we come back to this example in the following sections.

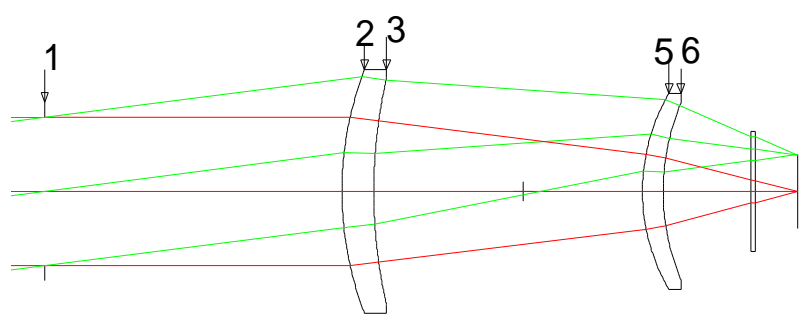

FIG. 1 IR imager with diamond-turned, diffractive element on surface 3 is shown.

\subsection{Tolerancing: Decentring from the diamond tool or laser beam relative to the axis of rotation}

In this case, the coordinate system of the cutting tool is parallel shifted by a misalignment $\Delta h$ with respect to the rotation axis (spindle axis) of the work piece by a constant amount, see Figure 2.

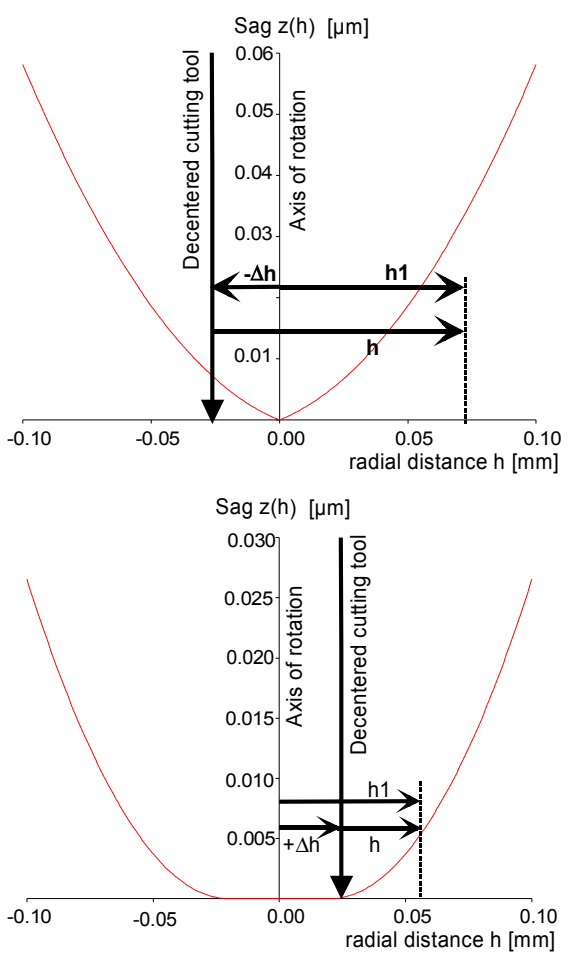

FIG. 2 For negative (top) and positive (bottom) shift $\Delta h= \pm 20 \mu \mathrm{m}$ due to translation Eq.(6) the resulting sag of the surface is plotted in closer proximity to the rotation axis. During manufacturing the tool moves in $+h$-direction.

\begin{tabular}{|l|l|l|l|l|}
\hline Surface & Radius & Thickness & Material & Dia \\
\hline 0 OBJ & Infinity & Infinity & Air & \\
\hline 1 STO & Infinity & 70.000 & Air & 35.0 \\
\hline 2 & 79.719 & 7.500 & ZnSe & \\
\hline 3 DOE & 120.410 & 35.250 & Air & \\
\hline 4 & Infinity & 28.000 & Air & \\
\hline 5 & 45.643 & 4.900 & Germanium & \\
\hline 6 & 53.858 & 20.650 & Air & \\
\hline 7 & Infinity & 1.000 & Germanium & \\
\hline 8 & Infinity & 9.985 & Air & \\
\hline
\end{tabular}

TABLE 1 This contains the data of the IR imager. The units are in $\mathrm{mm}$. The aperture stop is on surface 1 and has a diameter of $35.0 \mathrm{~mm}$. The field of view is $\pm 8.5^{\circ}$. The diffractive optical element (DOE) on surface 3 has the following coefficients according to Eq.(2): $c_{2}=-1.8381 E-04 ; c_{4}=6.1780 E-08 ; \lambda_{0}=0.010 \mathrm{~mm}$.

The actual radial distance $h_{1}$ is changed by the shift of $\Delta h$ and is given by:

$$
h_{1}=h+\Delta h
$$

with

$h_{1}$ Actual radial distance of the cutting tool

$h$ Target value of the radial distance

$\Delta h$ Shift between cutting tool and axis of rotation

This error produces a surface deformation and simultaneously a deformation of the diffractive structure. The shape of the surface will be deformed like shown in Figure 2 [4] and 
the diffractive part of the surface is changed like shown in Figure 3.
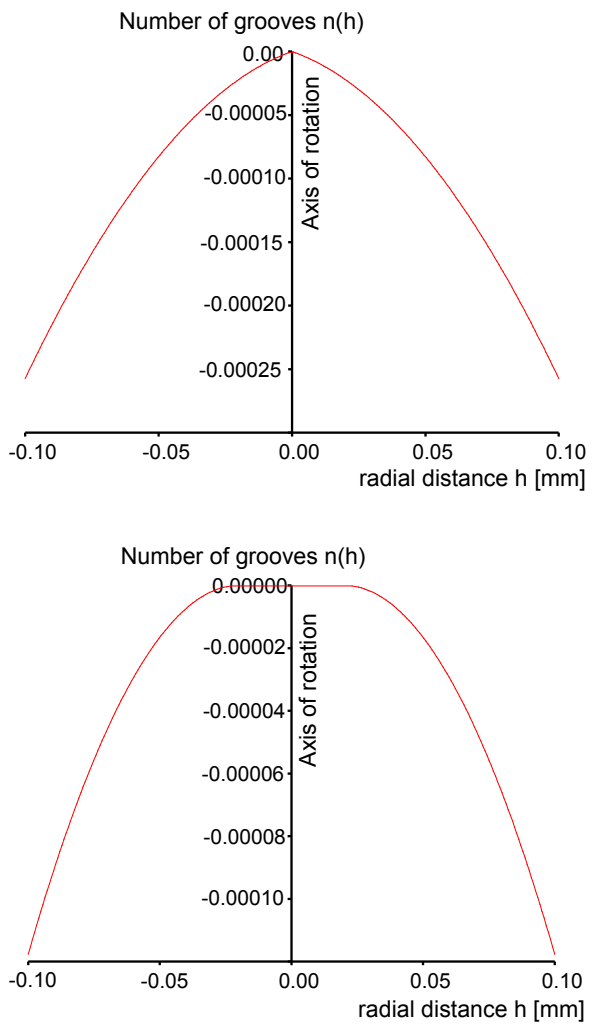

FIG. 3 For negative (top) and positive (bottom) shift $\Delta h= \pm 20 \mu \mathrm{m}$ due to translation Eq.(6) the resulting groove numbers Eq.(2) are plotted in closer proximity to the rotation axis. During manufacture the tool moves in $+\mathrm{h}$-direction.

\section{Example 3.2:}

On surface 3 in the lens system of Section 3.1 the shift of the tool is set to $\Delta h= \pm 20 \mu \mathrm{m}$ (Figures 2 and 3). Very similar shapes are seen with the surface shape in Figure 2 and with the number of the grooves in Figure 3. In order to show the shape in closer proximity to the surface vertex, the aperture of the surface in Figures 2 and 3 was limited strongly reduced from diameter 52.4 to $0.2 \mathrm{~mm}$. To assess the tolerances, at first the sag differences between the target surface and the toleranced surface are represented in Figure 4. Here the full $52.4 \mathrm{~mm}$ diameter of the surface is used. The vertices of the two figures are different, with the vertex being slightly rounded at the bottom and unrounded at the top. The shift error $\Delta h$ here results in a conical deformation between the target and actual surface, as Figure 4 shows. However, if the curves are continued further outwards, pronounced roundness also arises, which means that this error can not be treated as a pure conical error in every case.

Just like the sag differences between the target surface and the actual surface, the differences between the target groove number $\tilde{n}(h)$ and the actual groove number will be examined. Figure 5 shows the change in the groove number over the surface. Here, the deviation of the groove error from a cone already becomes visible within the active surface.
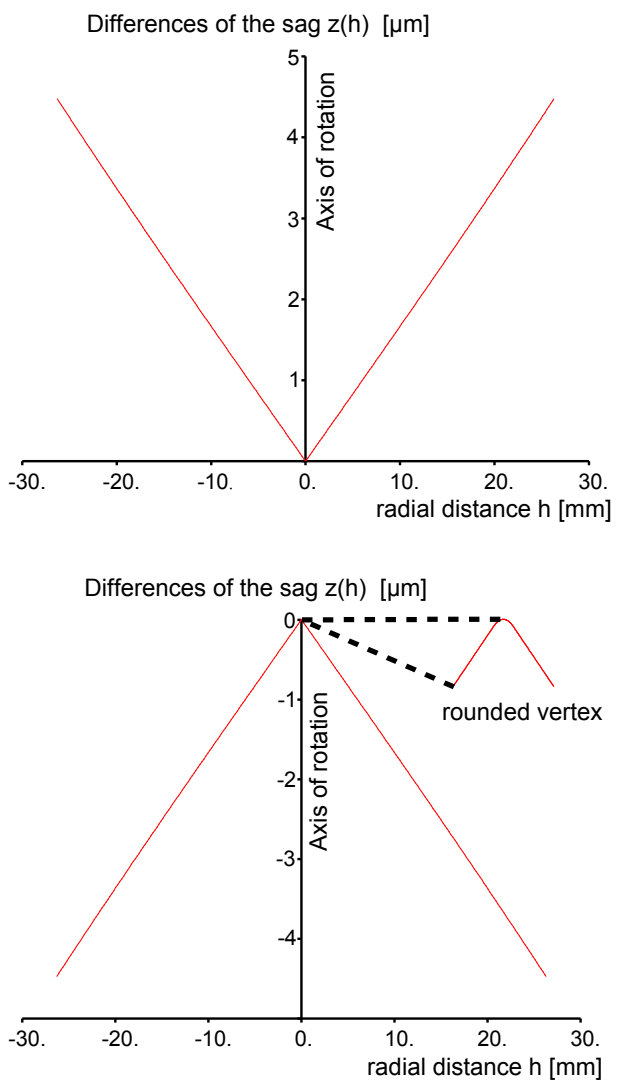

FIG. 4 For the tool shift of $\Delta h= \pm 20 \mu \mathrm{m}$ the differences between the target surface and actual surface for the full diameter of the lens are drawn here. Top: $-20 \mu \mathrm{m}$ shift; bottom: $+20 \mu \mathrm{m}$ shift.

We shall now consider the influence of a shift $\Delta h$ on the OPD by means of the analytic approximations Eq.(4) and Eq.(5), in order to better understand the conical figure of the resulting deformations in the Figures 4 and 5. According to Eqs.(1), (4) and (6), with the shift $\Delta h$ of the tool the surface figure error yields the following contribution to the OPD in the first order approximation and for small angles $\phi, i, i^{\prime}$ :

$$
\triangle O P D=\frac{\mathrm{n}-1}{\lambda}\left(\rho+2 b_{2}\right) \Delta h h
$$

This is a cone for $h>0$.

According to Eqs.(3) and (5), with a shift $\Delta h$ of the tool the following contribution to the OPD results from the error in the number of the grooves in the first order approximation:

$$
\triangle O P D=\frac{2 c_{2} \Delta h}{\lambda_{0}} h
$$

This is a cone for $h>0$ also.

This means: in the first order approximation with a constant shift of the tool, linear conical aberration of the OPD occurs according to Eq.(7) and Eq.(8) if the surface or the diffractive element has a focusing effect [6].

\subsection{Tolerancing: Variable shift between tool and spindle}

In addition to the constant shift from Section 3.2, slow variable and oscillating position errors also appear in practice [5] 

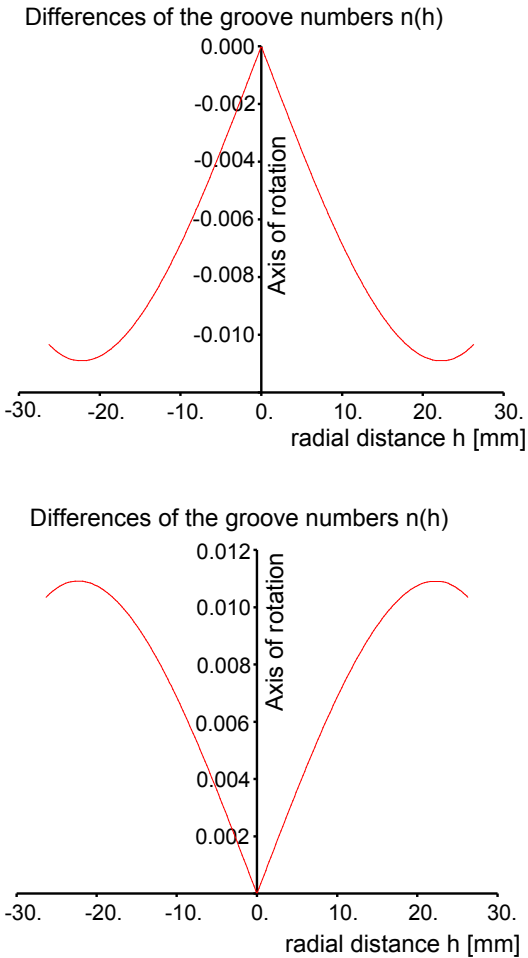

FIC. 5 This shows the differences between the target groove number and the actual groove number for a shift $\Delta h= \pm 20 \mu \mathrm{m}$ of the tool. Top: $-20 \mu \mathrm{m}$ shift; bottom: $+20 \mu \mathrm{m}$ shift.

caused by centripetal force or thermal effects during fabrication. In this case, the coordinate system of the tool is variably shifted relative to the rotation axis of the spindle due to an adjustment and a procedural error. Both the number of grooves and the shape of the surface are impaired by these position errors.

If $h$ is the radial distance from the axis of rotation of the target asphere and if the tool is shifted by $\Delta h\left(h_{1}\right)$ relative to the rotation axis, the approach is similar to Eq.(6). We can then write:

$$
h_{1}=h+\Delta h\left(h_{1}\right)
$$

with

$h_{1} \quad$ Actual radial distance from rotation axis

$h \quad$ Target radial distance

$\Delta h\left(h_{1}\right)$ Variable position error function of $h_{1}$

We expand the variable position error $\Delta h\left(h_{1}\right)$ in a power series and add a cosine-term to simulate oscillating errors. Thus the following form is assumed for the position error function :

$$
\begin{aligned}
\Delta h\left(h_{1}\right) & =a_{0}+a_{1} h_{1}+a_{2} h_{1}^{2}+a_{3} h_{1}^{3} \\
& +a_{4}\left(1-\cos \left(2 \pi a_{5} h_{1}\right)\right)
\end{aligned}
$$

with

$h_{1} \quad$ Actual radial distance from rotation axis

$a_{0} \quad$ Coefficient for constant decentring of the tool, analogous to $\Delta h$ in Eq.(6) $a_{1}, a_{2}, a_{3}$ Coefficients for higher orders

$a_{4} \quad$ Amplitude of the oscillating tool decentring

$a_{5} \quad$ Frequency of the oscillating tool decentring

With the notation used in Eq.(9) and Eq.(10) we can largely maintain the original form of the ray tracing modules for the tolerance calculation. We only have to enter simple extensions for the asphere and the diffractive element in the existing surface representations:

If the function originally used for the asphere Eq.(1) or for the diffractive element Eq.(2) is $f(h)$ here, then with the transformation Eq.(9) the new function is:

$$
f_{1}\left(h_{1}\right)=f\left(h_{1}-\Delta h\left(h_{1}\right)\right)=f(h)
$$

i. e. for the evaluation of the disturbed asphere or the impaired diffractive element, it is sufficient to implement the transformation Eq.(9) in the optical design program, while maintaining the function computation.

CodeV design software was used here. The partial derivatives of the function $f_{1}\left(h_{1}\right)$ are also needed for better performance in the user defined surface macro and in the diffractive element macro. These partial derivatives can be computed as follows. With the surface coordinates $x, x_{1}, y, y_{1}$ and $h=\sqrt{x^{2}+y^{2}}, h_{1}=\sqrt{x_{1}^{2}+y_{1}^{2}}$ it is:

$$
\begin{aligned}
\frac{\partial f_{1}\left(h_{1}\right)}{\partial x_{1}} & =\frac{\partial f}{\partial h} \cdot \frac{\partial h}{\partial h_{1}} \cdot \frac{\partial h_{1}}{\partial x_{1}} \\
& =\frac{\partial f}{\partial h} \cdot \frac{\partial h}{\partial x} \cdot \frac{\partial h}{\partial h_{1}} \quad \text { since } \quad \frac{\partial \mathrm{h}_{1}}{\partial \mathrm{x}_{1}}=\frac{\partial \mathrm{h}}{\partial \mathrm{x}} .
\end{aligned}
$$

The last factor must be supplemented in the calculating module of the user defined surface and the user defined diffractive element. From Eqs.(9) and (10) it follows:

$$
\frac{\partial h}{\partial h_{1}}=1-a_{1}-2 a_{2} h_{1}-3 a_{3} h_{1}^{2}-a_{4} \sin \left(2 \pi a_{5} h_{1}\right) 2 \pi a_{5}
$$

\section{Example 3.3:}

On surface 3 of the lens shown in Figure 1 the following deviations are assumed: $a_{0}=0.01 \mathrm{~mm}, a_{4}=0.003 \mathrm{~mm}, a_{5}=0.2$ lines per $\mathrm{mm}$.

Figure 6 shows the deviation of the sag. Figure 7 shows the deviation of the groove number and the related wavefront aberration. The resulting OPD for both the deviation of the sag and the groove number is shown in Figure 8 for the axial bundle and a field bundle of the IR Imager.

\subsection{Tolerancing: Tilt of the machine slide relative to the spindle}

A conical surface deformation results from a tilt of the tool's moving direction relative to the spindle axis (Figure 9). The deformation is rotationally symmetric and grows linearly with $h$. The slide tilt error can be simulated by a linear term in the sag of the asphere Eq.(1). With tilt $\alpha$ of the tool and ra- 

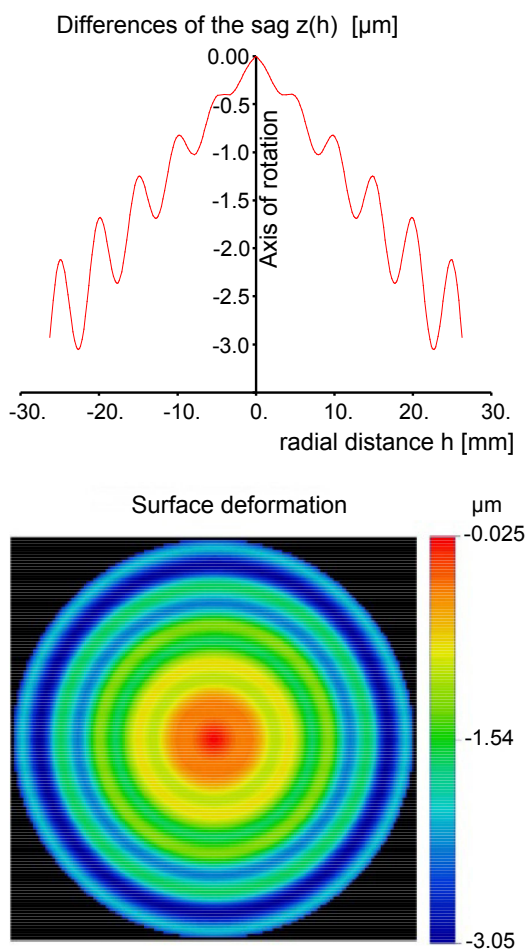

FIC. 6 Figure error of the diffractive surface 3 in example 3.3
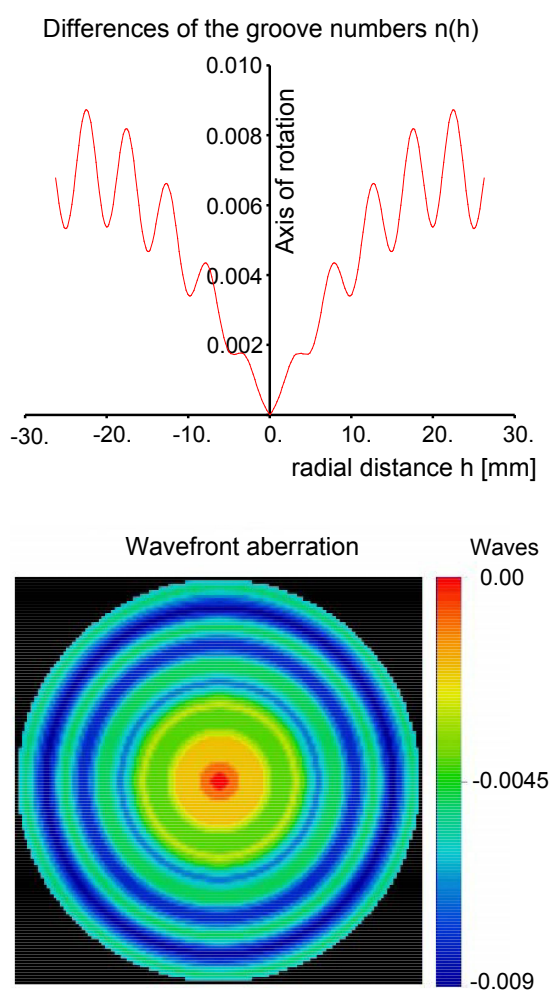

FIG. 7 Differences of the groove numbers between with and without tolerance in example 3.3. and the wavefront aberration of the diffractive part with this tolerance.

dial distance $h$, the deviation $\mathrm{t}$ of the sag is given by:

$$
t=-h \cdot \tan \alpha
$$

A comparison of the coefficients in the sag of the asphere Eq.(1) results in:

$$
b_{1}=-\tan \alpha
$$

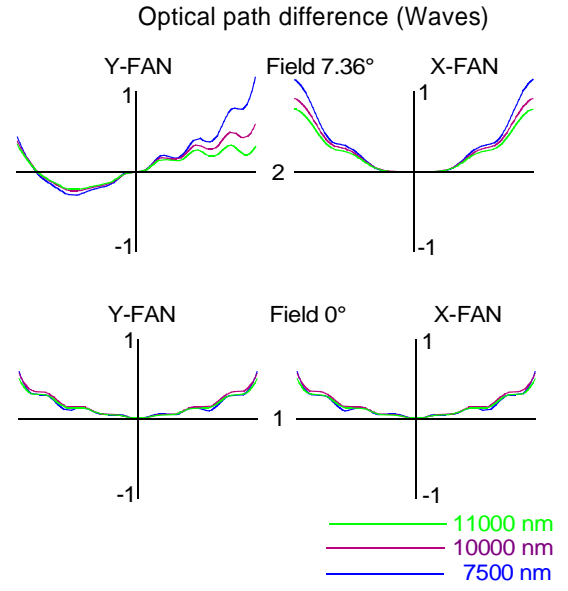

FIG. $8 O P D$ at $7500 \mathrm{~nm}, 10000 \mathrm{~nm}, 11000 \mathrm{~nm}$ for the field angle $0^{\circ}$ and $7.36^{\circ}$ in example 3.3 with the tolerances in Figure 6 and Figure 7 .

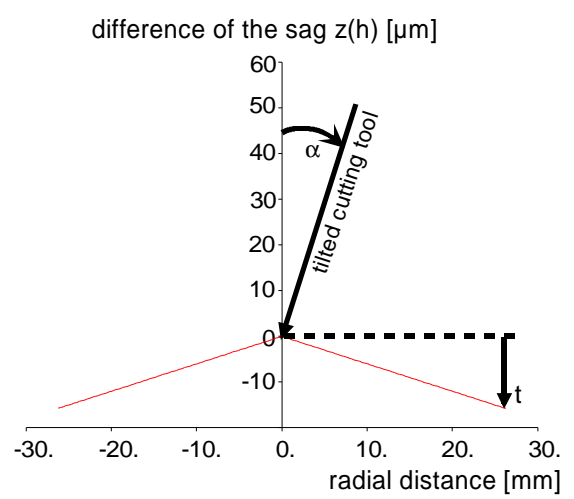

FIC. 9 This shows conical deformation of the sag with a slide tilt error. The red lines correspond to the deviation of the sag at $\alpha=2^{\prime}$ tilt of the slide with the production of surface 3 in example 3.1. This error is a pure surface deviation with no change in the diffractive structure.

\subsection{Tolerancing: Periodic structures by thermal effects or tool wear}

Ripples in both the refractive and diffractive structure are examined in Section 3.3. Periodic structures (ripples) on the surface can also result from uneven cooling of the tool or the carrier during fabrication or also from uneven cutting tool wear. Such effects result in a pure surface deformation, while the grooves of the diffractive element remain in position. These ripples are described by the following parameters [4]:

- Amplitude of the periodic deviation

- Number of the oscillations over the surface

- The phase of the periodic function

- Exponent of the periodic function

- Damping factor of the amplitude

For the numerical simulation of this additional ripples Eq.(1) is extended by a damped sinusoidal function of a higher- 
order. The following sag $z_{1}(h)$ results:

$$
z_{1}(h)=z(h)+A_{1} e^{D h} \cdot \sin ^{\mathcal{S}}\left(B_{1} \pi h+\phi_{1}\right)
$$

with

$z(h)$ Sag according to Equation (1)

h Radial distance

$A_{1} \quad$ Scale factor

D Damping parameter of the amplitude

$s \quad$ Exponent of the sine (even-numbered)

$B_{1} \quad$ Frequency in oscillations per $\mathrm{mm}$.

$\phi_{1} \quad$ Phase

\section{Example 3.5:}

On surface 3 in Figure 1 the deviations shown in Figure 10 are assumed. The resulting wavefront aberration is shown in Figure 11.
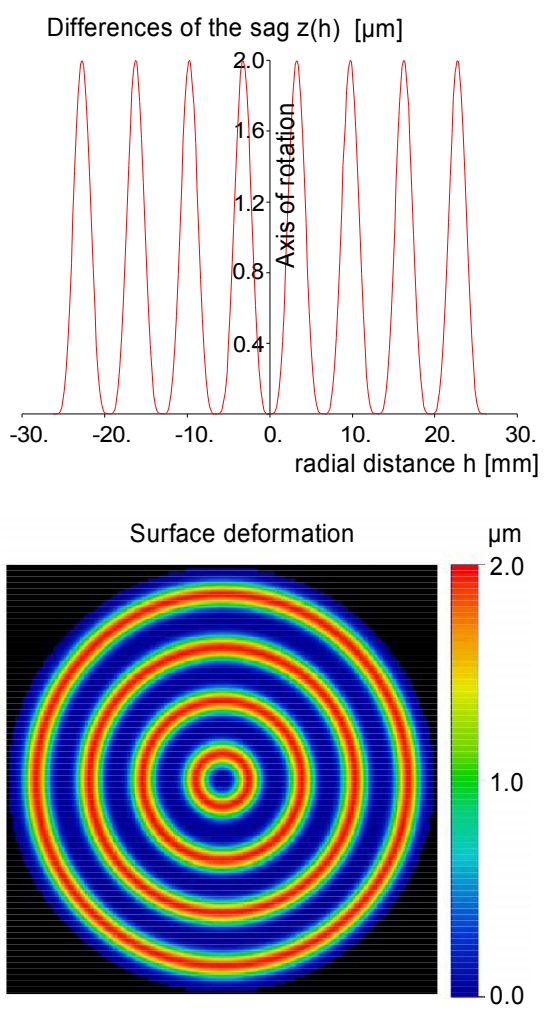

FIC. 10 Deviations between the target and actual surface with the following parameters in Eq.(17): $A_{1}=0.002 ; D=0 ; s=4 ; B_{1}=0.1538 \mathrm{~mm}^{-1}$.

\subsection{Tolerancing: Vibration of the axis of rotation, "spindle star"}

Vibrations of the spindle perpendicular to the workpiece can result in azimuthal ripples on the surface. In the interferogram of the surface a star is seen, the "spindles star" [4]-[6]. However, a "spindle star" is more indicative of a machine defect

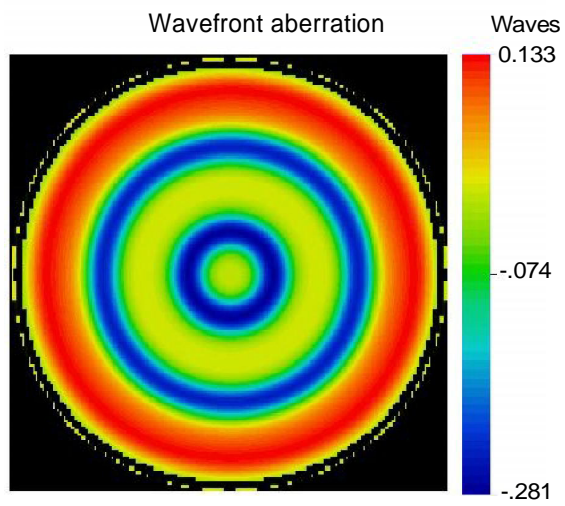

FIG. 11 Resulting wavefront aberration in the axial bundle with the tolerances is shown in Figure 10.

than of a reasonably tolerable size. If it occurs, its magnitude may vary. It does not occur in a reasonable turning process.

To simulate the "spindle star" we start from Eq.(17) and extend it by $\sin ^{2}$-term in the azimuth. The variable amplitude of the $\sin ^{2}$ is expanded in the first terms of a power series. This describes the "spindle star" with the following sag:

$$
z_{2}(h)=z_{1}(h)+\left(A_{0}+A_{2} h\right) \sin ^{2}\left(B_{2} \frac{\phi}{2}\right)
$$

with

$z_{1}(h) \quad$ Sag of Eq.(17)

$A_{0}+A_{2} h \quad$ Variable amplitude factor

$B_{2} \quad$ Number of the spokes of the "spindle star"

$\phi \quad$ Azimuth in radians

Example 3.6:

Figure 12 shows a "spindle star" with 17 spokes on surface 3 of the lens in Figure 1.

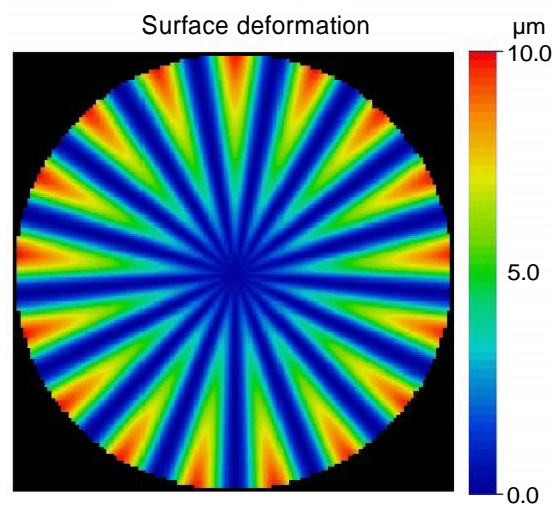

FIG. 12 Surface error "spindle star" caused by vibrations with $A_{0}=0, A_{2}=0.00038$, $B_{2}=17$. The star has an amplitude of $10 \mu \mathrm{m}$ at $h=26.2 \mathrm{~mm}$ and has 17 spokes. The 'spindle star' should not occur in a reasonable turning process. 


\subsection{Remark}

In Section 3 most notably rotational symmetric errors are considered. E.g. through pressure caused when mounting the workpiece, surface deformations like curvature error, cylindrical error, trefoil error on the finished lens can arise [4]. Tolerances for such errors are standard tolerances, which are very important, but they are not considered here.

\section{CONCLUSIONS}

This paper examines the characteristic errors and their effects on aberrations, which arise with single point diamond turning. Similar errors also arise in laser plotting of diffractive elements. The turning process for optical surfaces and diffractive elements on aspheric surfaces is considered. Compared with other manufacturing methods, it is possible to find typical error types. Among other things, the tool decentring and its tilting to the axis of rotation can be toleranced here. For these special errors the simultaneous effect of the diffractive and the refractive part of the surface error is considered with regard to the aberrations. Standard tolerances of curvature error or cylindrical error are also present with the SPDT.

\section{ACKN OWLED GMENTS}

This work has been supported by the German Federal Ministry of Research and Technology (BMBF) with the support number FKZ 13N8478 under the project name: 'HYBROS;
Neuartige hybride Optik-Systeme'. Project bearer: VDI Technologiezentrum $\mathrm{GmbH}$.

I am grateful to Dr. J. Ruoff, Dr. Markus Seesselberg and Dr. $\mathrm{H}$. Gross for a thorough review of the text. I thank C. Beder and Dr. M. Pollmann for their discussions about their practical experiences.

\section{References}

[1] J. P. Schaefer, ThB1.pdf, Vancoover IODC (2006).

[2] Gabor Erdei, Gabor Szarvas, Emöke Lörincz, "Tolerancing surface accuracy of aspheric lenses used for imaging purposes" Proc. of SPIE 5249, 718-728 (2004).

[3] J. P. Marioge, S. Slansky, "EFFET DES DÉFAUTS DE FORME ET DES ODULATIONS DES SURFACES OPTIQUES SUR LA QUALITÉ DES IMAGES, Effect of figure and waviness on image quality" J. Optics (Paris) 14, 189-198 (1983).

[4] R. C. Juergens, R. Hamilton Shepard III, John P. Schaefer, "Simulation of single point diamond turning fabrication process errors" Proc. of SPIE 5174, 93-104 (2003).

[5] R. Freimann, "Aberrations of axially symmetric diffractive optical elements in relation to their fabrication inaccuracies" Optik 111, 485-492 (2000).

[6] S. Reichelt, M. Daffner, H. J. Tiziani, R. Freimann, “Wavefront aberrations of rotationally symmetric CGHs fabricated by a polar coordinate laser plotter" J. Mod. Optic 49, 1069-1087 (2002).

[7] CodeV trademark of Optical Research Associates. 